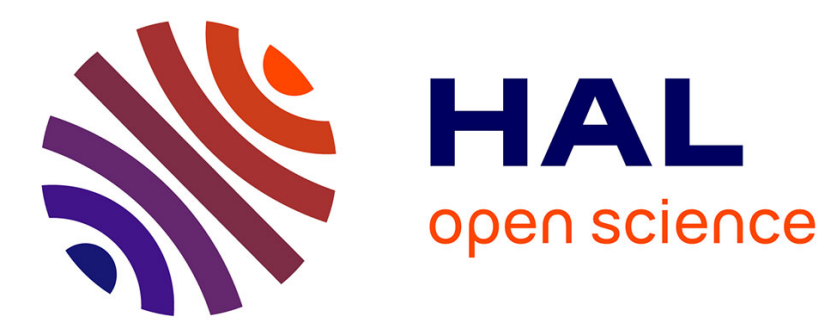

\title{
Cooling by Baroclinic Acoustic Streaming
}

Guillaume Michel, Christophe Gissinger

\section{To cite this version:}

Guillaume Michel, Christophe Gissinger. Cooling by Baroclinic Acoustic Streaming. Physical Review Applied, 2021, 16, pp.L051003. 10.1103/PhysRevApplied.16.L051003 . hal-03437452

\section{HAL Id: hal-03437452 \\ https://hal.science/hal-03437452}

Submitted on 19 Nov 2021

HAL is a multi-disciplinary open access archive for the deposit and dissemination of scientific research documents, whether they are published or not. The documents may come from teaching and research institutions in France or abroad, or from public or private research centers.
L'archive ouverte pluridisciplinaire HAL, est destinée au dépôt et à la diffusion de documents scientifiques de niveau recherche, publiés ou non, émanant des établissements d'enseignement et de recherche français ou étrangers, des laboratoires publics ou privés. 


\title{
Cooling by baroclinic acoustic streaming
}

\author{
Guillaume Michel ${ }^{1 *}$ and Christophe Gissinger ${ }^{2,3}$ \\ ${ }^{1}$ Sorbonne Université, CNRS, Institut Jean Le Rond d'Alembert, F-75005 Paris, France \\ 2 Laboratoire de Physique de l'Ecole normale supérieure, ENS, Université PSL, \\ CNRS, Sorbonne Université, Université de Paris, Paris, France and \\ 3 Institut Universitaire de France (IUF)
}

(Dated: November 15, 2021)

\begin{abstract}
In the absence of natural convection, efficient heat transfers rely on externally forced flows. Generating such flows with acoustic waves rather than mechanical fans would enable remote locations to be cooled using virtually infinite lifetime transducers. This outlook is reinforced by the recent discovery that standing acoustic waves drive streaming flows of much higher velocities if the background medium is inhomogeneous. This regime of streaming is investigated experimentally in a cavity filled with stably-stratified air in which horizontal sound waves are found to significantly enhance heat transfers. The additional heat flux scales as the square of the input acoustic power for low amplitude waves and increases with the air stratification. These two features qualitatively match theoretical predictions, although corrections possibly ascribed to gravity are observed.
\end{abstract}

Introduction. - The generation of streaming flows by acoustic waves in homogeneous fluids requires a dissipation mechanism [1]. Close to solid boundaries, viscosity generates oscillating boundary layers that can locally force a mean flow: this so-called Rayleigh streaming is used in microfluidics to improve the efficiency of catalytic solid phases [2] or to mix chemicals [3]. In the bulk, the attenuation of acoustic beams forces jets, a phenomenon referred to as quartz wind. As reviewed in Ref. [4], most studies investigating the effect of acoustic waves on heat transfers typically involve generating such progressive ultrasonic beams with a few hundred watts in a liquid to trigger cavitation and/or drive forced convection.

Over the last few decades, a very different picture has emerged for acoustic streaming in inhomogeneous fluids. Experiments and numerical simulations with standing waves started investigating this regime and reported radically different streaming patterns, in some cases several orders of magnitude faster than the corresponding ones in homogeneous fluids [5-10]. This regime of streaming was rationalized theoretically for gases by Chini et al. [11] and termed baroclinic acoustic streaming. Its most noticeable feature is that the acoustic wave vorticity required to drive streaming no longer results from dissipation but is a consequence of baroclinicity, an inviscid process. This mechanism can be inferred from the curl of the linearized Euler equation describing the leading-order acoustic wave dynamics:

$$
\nabla \times\left(\rho \frac{\partial \mathbf{u}}{\partial t}=-\nabla p\right) \Rightarrow \frac{\partial(\nabla \times \mathbf{u})}{\partial t}=\frac{(\nabla \rho) \times(\nabla p)}{\rho^{2}},
$$

where $\rho$ is the time-averaged density, $\mathbf{u}$ is the acoustic wave velocity and $p$ is the acoustic wave pressure. Whereas inviscid acoustic waves in homogeneous fluids have no vorticity, Eq. (1) demonstrates that this no longer holds in the presence of a background stratification as a result of baroclinic production of vorticity.
The acoustic force density driving these streaming flows has then been derived in the general case of liquids, for both density and compressibility inhomogeneities [12]. Since the properties of the acoustic modes (frequency and eigenfunction) depend on these background fields, a subtle two-way coupling takes place: the fast acoustic waves generate a slowly varying mean flow that advects inhomogeneities and feeds back on the acoustic waves.

Several applications of this regime of streaming are already documented. It can significantly improve the efficiency of high-intensity discharge lamps, in which the mean temperature varies by thousands of degrees between the center and the walls [10]. In microfluidics, it affects the acoustically-induced mixing of different fluids [13]. According to an experiment with water in a microchannel, a local temperature fluctuation of $\sim 0.2 \mathrm{~K}$ is sufficient to distort the streaming pattern observed in a homogeneous fluid [14]. The baroclinic streaming flow in a stratified gas enclosed between two horizontal walls is characterized in Ref. [15] and quantitatively fits previous numerical simulations (see also Ref. [16] in the limit of small temperature gradients). It suggests a new procedure based on stationary waves to acoustically enhance heat transfers in the absence of natural convection, e.g. for stably stratified or zero-gravity environments $[15,17]$. However, several thermal properties of this system, crucial for assessing the potential of acoustic streaming for practical applications such as cooling electronics aboard spacecraft, are still missing.

In this letter, we show that baroclinic streaming can significantly enhance heat transfers and characterize this cooling effect as a function of both the acoustic wave properties and the gas stratification. The results are compared with theoretical predictions from dissipation-based streaming [18] and baroclinic streaming in Ref. [15].

* email: guillaume.michel@upmc.fr 


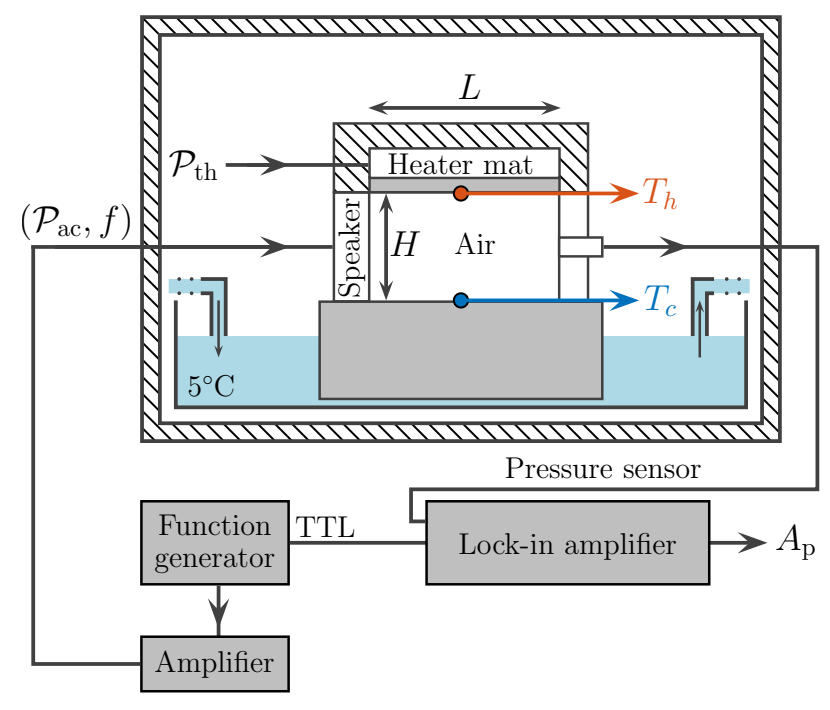

FIG. 1. Experimental setup. A speaker, driven by a harmonic signal of frequency $f$ and power $\mathcal{P}_{\text {ac }}$, generates an acoustic wave of local amplitude $A_{p}$ measured at the opposite end. The cavity of height $H$ and length $L$ is heated at the top (power $\mathcal{P}_{\text {th }}$, temperature $T_{h}$ ) and cooled at the bottom (temperature $\left.T_{c}\right)$. The width $\ell$ is not displayed. Gray areas represent dural samples and hatched areas represent thermal insulators.

Experiment. - The experiment sketched in Fig. 1 consists of a cavity of length $L=10 \mathrm{~cm}$, height $H=$ $5.0 \mathrm{~cm}$ and width $\ell=3.0 \mathrm{~cm}$ filled with air at atmospheric pressure. Heat is injected at the top wall at a rate $\mathcal{P}_{\text {th }}$ through an RS Pro silicon heater mat fixed on a metallic plate. The bottom wall is a block of dural laterally cooled by means of water at $5^{\circ} \mathrm{C}$ provided by a Lauda Master thermostat. The top and bottom temperatures at the center of these boundaries, measured by type $\mathrm{K}$ thermocouples and Keithley 2700 multimeters, are denoted by $T_{h}$ and $T_{c}$. A Visaton SC-5 tweeter subject to an input sinusoidal signal of frequency $f$ and power $\mathcal{P}_{\text {ac }}$ constitutes one lateral wall of this cavity, at the opposite end of which a PCB 103A02 sensor is fixed. The signal from this sensor, processed by a PCB 480E09 signal conditioner and a Standford Research Systems SR 830 lock-in amplifier synchronized on the frequency $f$, yields an accurate measurement of the local amplitude $A_{\mathrm{p}}$ of the oscillating pressure field. The remaining boundaries are $10 \mathrm{~mm}$ thick PEEK samples, this material being chosen for its thermal insulation properties and heat resistance. The entire setup is surrounded by thermal insulators, and the room itself is air conditioned to further reduce the daily temperature oscillations. Stationary states are reached after a transient of one hour corresponding to thermal diffusion in the lateral PEEK boundaries. Measurements of $\left(T_{h}, T_{c}, A_{\mathrm{p}}\right)$ as a function of the input parameters $\left(\mathcal{P}_{\text {th }}, \mathcal{P}_{\text {ac }}, f\right)$ are performed during two hours once stationary states are achieved and repeated between 3 and 9 times for statistics, the data reported in this letter summarizing around 2000 hours of acquisition.

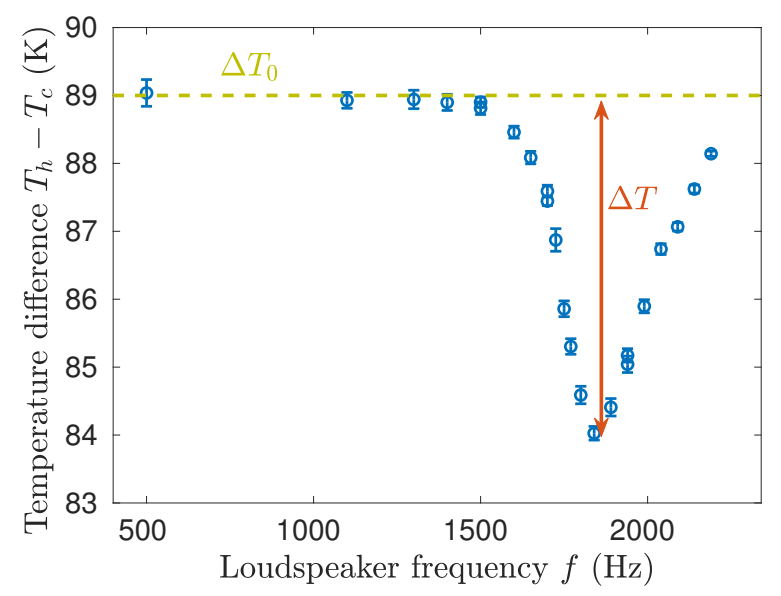

FIG. 2. Top to bottom temperature difference $T_{h}-T_{c}$ as a function of the forcing frequency $f$ for $\mathcal{P}_{\text {ac }}=0.5 \mathrm{~W}$ and $\mathcal{P}_{\text {th }}=8.8 \mathrm{~W}$. The corresponding temperature stratification $\Delta T_{0}$ and streaming temperature drop $\Delta T$ are also reported.

A first run is performed for various frequencies $f$, the injected heat $\mathcal{P}_{\text {th }}=8.8 \mathrm{~W}$ and input power in the loudspeaker $\mathcal{P}_{\mathrm{ac}}=0.5 \mathrm{~W}$ being constant. The top to bottom temperature difference, reported in Fig. 2, reaches a minimum at around $1.84 \mathrm{kHz}$. This frequency corresponds to the first acoustic mode of the cavity, which would read $a /(2 L)$ for a homogeneous gas, with $a$ the speed of sound. Under the assumption of a linear temperature stratification and a thin cavity, this eigenvalue problem still allows a simple analytic solution [15]:

$$
f_{0}=\frac{a_{\mathrm{c}}}{2 L} \sqrt{1+\left(\frac{T_{h}-T_{c}}{2 T_{c}}\right)},
$$

where the sound speed, no longer uniform, is $a_{\mathrm{c}}$ at $T_{c}$. With $a_{\mathrm{c}}=335 \mathrm{~m} \cdot \mathrm{s}^{-1}$ and the mean values for this run $T_{c}=16^{\circ} \mathrm{C}$ and $T_{h}=105^{\circ} \mathrm{C}$, eq. (2) yields $f_{0}=1.8 \mathrm{kHz}$. From Fig. 2, we define the temperature stratification $\Delta T_{0}=89 \mathrm{~K}$ as the low frequency limit of $T_{h}-T_{c}$ and the streaming temperature drop $\Delta T=5 \mathrm{~K}$ as the variation range of $T_{h}-T_{c}$. Note that $\Delta T_{0}$ corresponds to the top to bottom temperature difference in the absence of acoustic waves, when the input power in the speaker is directly converted to heat. The remainder of this investigation details how $\Delta T_{0}$ and $\Delta T$ depend on $\mathcal{P}_{\text {th }}$ and $\mathcal{P}_{\text {ac }}$.

The procedure leading to Fig. 2 is repeated for various input acoustic power $\mathcal{P}_{\text {ac }}$ at fixed heating $\mathcal{P}_{\text {th }}=8.8 \mathrm{~W}$, the resulting relative temperature drop $\Delta T / \Delta T_{0}$ being reported in Fig. 3. A quadratic function $0.4 \mathcal{P}_{\text {ac }}^{2}$ fits the data in the limit $\Delta T / \Delta T_{0} \rightarrow 0$, while overestimating those obtained at high acoustic power.

Similarly, the influence of the dimensionless temperature stratification $\Gamma=\Delta T_{0} / T_{c}$ is investigated with runs performed for varying $\mathcal{P}_{\text {th }}$ at fixed $\mathcal{P}_{\mathrm{ac}}=0.5 \mathrm{~W}$. The relative temperature drop, reported in Fig. 4, increases with $\Gamma$ and can be approximated by $4 \times 10^{8} F(\Gamma)$ with $F$ a 


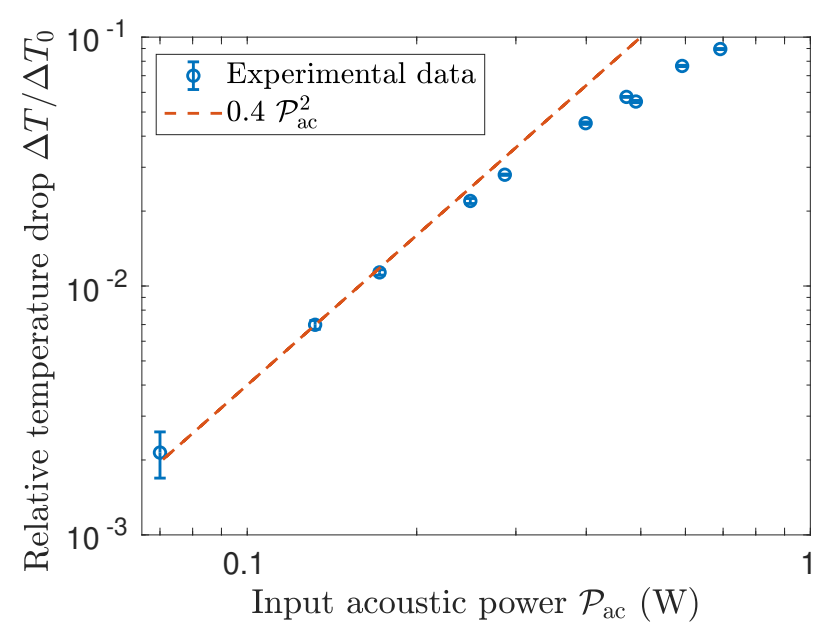

FIG. 3. Relative temperature drop $\Delta T / \Delta T_{0}$ as a function of the injected power in the loudspeaker $\mathcal{P}_{\text {ac }}$ for $\mathcal{P}_{\text {th }}=8.8 \mathrm{~W}$.

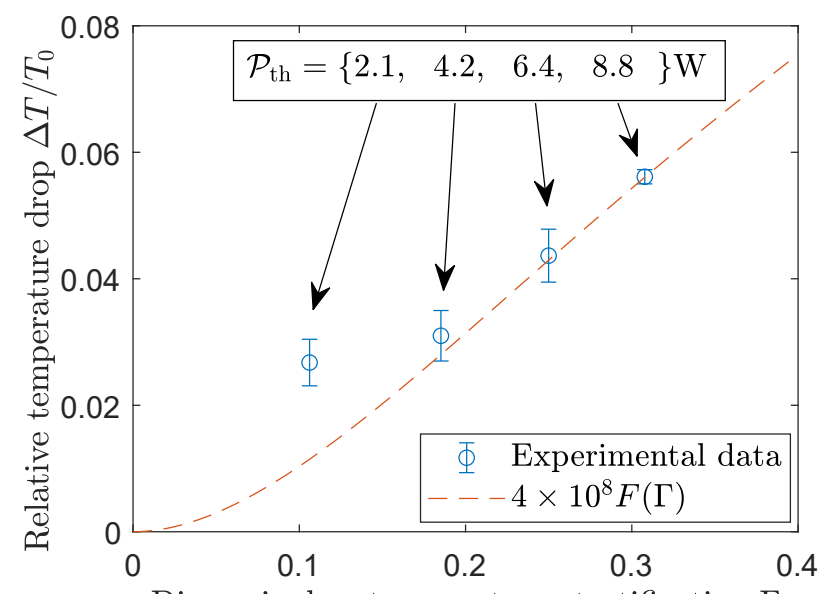

Dimensionless temperature stratification $\Gamma$

FIG. 4. Relative temperature drop $\Delta T / \Delta T_{0}$ as a function of the dimensionless temperature stratification $\Gamma=\Delta T_{0} / T_{c}$ for fixed power supplied to the loudspeaker $\mathcal{P}_{\mathrm{ac}}=0.5 \mathrm{~W}$ and various heating $\mathcal{P}_{\text {th }}$. Note that $\Delta T / \Delta T_{0}$ would be constant if the streaming flow were independent of $\Gamma$.

theoretical function with no fit parameter detailed in the Appendix. The amplitude $A_{\mathrm{p}}$ of the oscillating pressure field is found to be $(25 \pm 2) \mathrm{Pa}$. It provides the typical acoustic velocity, $U \sim A_{\mathrm{p}} /\left(\rho a_{\mathrm{c}}\right) \sim 6 \mathrm{~cm} \cdot \mathrm{s}^{-1}$, with $\rho$ the air density.

Overall, these results demonstrate that, in a stratified gas, a significant temperature drop can be obtained by forcing a standing wave at resonance even for acoustic powers $\mathcal{P}_{\text {ac }}<1 \mathrm{~W}$ orders of magnitude below the ones typically used for cooling in liquids (a few hundred watts [4]). This cooling is found to increase with both the acoustic power and the thermal stratification.

\begin{tabular}{|c|c|}
\hline Dimensional parameters & Assumed value \\
\hline Sound speed $a_{\mathrm{c}}\left(\right.$ at $\left.T_{c}\right)$ & $335 \mathrm{~m} \cdot \mathrm{s}^{-1}$ \\
\hline Typical acoustic velocity $U$ & $6 \mathrm{~cm} \cdot \mathrm{s}^{-1}$ \\
\hline Horizontal wave number $k$ & $\pi / L=31 \mathrm{~m}^{-1}$ \\
\hline Height of the cavity $H$ & $50 \mathrm{~mm}$ \\
\hline Air density $\rho$ (at $T_{c}$ ) & $1.25 \mathrm{~kg} \cdot \mathrm{m}^{-1}$ \\
\hline Dynamic viscosity $\mu$ (indep. of $T$ ) & $18 \mu \mathrm{Pa} \cdot \mathrm{s}$ \\
\hline Thermal conductivity $\kappa$ (indep. of $T$ ) & $28 \mathrm{~mW} \cdot \mathrm{K}^{-1}$ \\
\hline Isobaric specific heat $c_{p}$ (indep. of $T$ ) & $1.0 \mathrm{~kJ} \cdot \mathrm{kg}^{-1} \cdot \mathrm{K}^{-1}$ \\
\hline
\end{tabular}

TABLE I. Dimensional parameters used to model this experiment.

\begin{tabular}{|c|c|c|}
\hline Dimensionless parameters & Definition & Value \\
\hline Inverse Strouhal number $\varepsilon$ & $U / a_{\mathrm{c}}$ & $1.8 \times 10^{-4}$ \\
\hline Aspect ratio $h$ & $k H / \sqrt{\varepsilon}$ & $1.2 \times 10^{2}$ \\
\hline Streaming Reynolds number $R e_{s}$ & $\varepsilon \rho U /(k \mu)$ & $2.4 \times 10^{-2}$ \\
\hline Streaming Peclet number $P e_{s}$ & $\varepsilon \rho c_{p} U /(k \kappa)$ & $1.5 \times 10^{-2}$ \\
\hline Temperature gradient $\Gamma$ & $\Delta T_{0} / T_{c}$ & $0.1 \leqslant \Gamma \leqslant 0.3$ \\
\hline
\end{tabular}

TABLE II. Dimensionless parameters describing the streaming flow.

Discussion. - These results are compared with theoretical predictions based on Rayleigh streaming [18] and baroclinic streaming [15]. Both models apply to an ideal gas enclosed in a thin cavity and subject to the following main assumptions: the dynamics is two-dimensional, the top and bottom walls are at fixed temperature, parameters such as $\mu, \kappa$ and $c_{p}$ are assumed independent of temperature (see Tab. I) and, most importantly, gravity is neglected. Although this last assumption precludes any quantitative comparison with the present experiment in which gravity acts as a strong restoring force, it is of interest to investigate if the associated scaling laws hold.

From the dimensional parameters used to model this experiment and listed in Tab. I, several dimensionless numbers can be defined, see Tab. II. The multiple scales theory of baroclinic streaming expands the various fields as series of $\varepsilon \ll 1$ and scales $h, R e_{s}, P e_{s}$ and $\Gamma$ as $O(1)$. The smallness of $\varepsilon$ in this experiment supports such multiple scales analysis. In the limit $\Delta T \ll \Delta T_{0}$ where the background density field can be approximated by the one at rest, the constant power and constant temperature boundary conditions become equivalent and the following temperature drop is derived:

$$
\frac{\Delta T}{\Delta T_{0}}=F(\Gamma)\left(A^{2} R e_{s} P e_{s} h^{4}\right)^{2},
$$

where $A$ is the dimensionless amplitude of the wave, assumed of $O(1)$ in the analysis and related to $A_{\mathrm{p}}$ through

$$
A_{\mathrm{p}}=\frac{\varepsilon p_{\mathrm{atm}} A \gamma}{\sqrt{\pi\left(1+\frac{\Gamma}{2}\right)}},
$$


with $p_{\text {atm }}=10^{5} \mathrm{~Pa}$ the ambient pressure and $\gamma=1.4$ the specific heat ratio.

It is instructive to compare eq. (3) with the temperature drop that would be achieved by forced convection with a fan of fixed rotation rate, $\Delta T \propto \Delta T_{0}$ : baroclinic acoustic streaming is such that $\Delta T \propto F(\Gamma) \Delta T_{0}$ with $F(\Gamma)$ an increasing function in the range $\Gamma \in[0,1.05]$, evidencing that increasing the temperature stratification $\Delta T_{0}$ also affects the streaming flow. With the dimensionless parameters listed in Tab. II, an arbitrary constant amplitude $A=0.55$ makes the right hand sides of eq. (3) become $4 \times 10^{8} F(\Gamma)$, corresponding to the dashed line plotted in Fig. 4 that fits our data for $\Gamma \geqslant 0.2$. Inferring $A$ from (4) with $\Gamma \simeq 0.2$ and $A_{\mathrm{p}}=25 \mathrm{~Pa}$ instead leads to $A \simeq 1.8$ that overestimates the temperature drop.

In comparison, the temperature drop resulting from Rayleigh streaming driven by dissipation in the boundary layers and dominant for homogeneous fluids is [18]

$$
\frac{(\Delta T)_{R}}{\Delta T_{0}}=6.2 \times 10^{-5}\left(\varepsilon A^{2} P e_{s} h^{2}\right)^{2} .
$$

With $A \sim 2$, the streaming temperature $\operatorname{drop}(\Delta T)_{R} \sim$ $10^{-6} \Delta T_{0}$ is negligible. This confirms that dissipationbased streaming is irrelevant in the presence of large temperature inhomogeneities.

Note that the scaling $\Delta T / \Delta T_{0} \propto \mathcal{P}_{\text {ac }}^{2}$ observed in Fig. 3 as $\mathcal{P}_{\text {ac }} \rightarrow 0$ is consistent with both Rayleigh and baroclinic streaming. In this limit, the two theories predict that both the relative temperature drop and the power transferred by the waves to the streaming flow scale as $A^{4}$. The injected acoustic power $\mathcal{P}_{\text {ac }}$ is then mainly balanced by the dissipation of energy in the oscillating boundary layers (proportional to $A^{2}$ ), hence $A \propto \sqrt{\mathcal{P}_{\text {ac }}}$ and $\Delta T / \Delta T_{0} \propto A^{4} \propto \mathcal{P}_{\text {ac }}^{2}$.

Conclusion. - Acoustic streaming resulting from inhomogeneities in the mean density field rather than dissipation processes recently emerged as an efficient mechanism to drive mean flows. Such setups typically stem from the use of different liquids $[12,13]$ or from large temperature fluctuations in a gas $[5-10,19]$. This affects not only the pattern of the streaming flow, but also its velocity. Indeed, whereas Rayleigh streaming is of velocity $O\left(U^{2} / a\right)$, with $U$ the typical acoustic velocity and $a$ the sound speed, baroclinic streaming in strongly stratified systems can be $O(U)$ [15]. Such strong streaming flow could enhance diffusive heat transfers observed in stably-stratified gas on earth or close to hot electronic devices aboard spacecraft. The present experiment confirms this potential, by decreasing the temperature difference across a stably-stratified gas by almost $10^{\circ} \mathrm{C}$ with low-power stationary acoustic waves $\left(\mathcal{P}_{\text {ac }}<1 \mathrm{~W}\right)$, in qualitative agreement with theoretical predictions [15] in which gravity is neglected, and several orders of magnitude above estimates based on Rayleigh streaming. This outlook is even more promising since the temperature drop increases more than linearly with the temperature difference, as measured by the right hand side of eq. (3): since $F(1) \simeq 2.2 F(0.32)$, a setup with $\left\{T_{c}=-62^{\circ} \mathrm{C}, T_{h}=150^{\circ} \mathrm{C}\right\}$ should for instance be associated with a relative temperature drop $\Delta T / T_{0} 120 \%$ higher than the one described Fig. 2 with $\left\{T_{c}=16^{\circ} \mathrm{C}, T_{h}=103^{\circ} \mathrm{C}\right\}$.

At least two perspectives emerge from this work. First, the experimental investigation of more intense acoustic waves, associated with streaming Reynolds numbers $R e_{s} \gg 1$ for which the streaming flow would presumably involve jets and/or turbulence [1]. The theoretical approach of such setups remains challenging and requires numerical simulations accounting for two-way coupling between the acoustic waves and the streaming flow $[11,15]$. Second, similar large-amplitude streaming flows should be expected wherever stationary acoustic waves develop in inhomogeneous fluids, as for instance in thermoacoustic devices [20] and in acoustically-trapped spherical plasma bulbs [16, 21].

This work is supported by CNES. CG acknowledges financial support from the French program 'JCJC' managed by Agence Nationale de la Recherche (Grant ANR19-CE30-0025-01).

Appendix In this appendix is derived the function $F(\Gamma)$ introduced in Eq. (3) from the multiple scales model of Ref. [15]. Following their notations, the dimensionless mean temperature field $\bar{\Theta}_{0}(x, y, \Gamma)$ is a solution of their equation (4.8),

$$
(1+\Gamma y) \partial_{\text {yyyyy }} \bar{\Theta}_{0}+3 \Gamma \partial_{\text {yyyy }} \bar{\Theta}_{0}=-\frac{2 A^{2} \Gamma^{2} R e_{s} P e_{s} h^{4}}{\pi(2+\Gamma)^{2}} \cos (2 x),
$$

complemented with six boundary conditions, setting to zero $\bar{\Theta}_{0}, \partial_{y y} \bar{\Theta}_{0}$ and $\partial_{y y y} \bar{\Theta}_{0}$ at $y=0$ and $y=1$ (whatever $x)$. The solution is found to be

$$
\bar{\Theta}_{0}(x, y, \Gamma)=-\frac{2 A^{2} \Gamma^{2} R e_{s} P e_{s} h^{4}}{\pi(2+\Gamma)^{2}} G_{\Gamma}(y) \cos (2 x),
$$

where $G_{\Gamma}(y)$ is only reported in Ref. [15] for $\Gamma=1$. Here, $G_{\Gamma}(y)$ is computed with Mathematica and reads

$$
G_{\Gamma}(y)=\frac{A+B+C}{D},
$$

where

$$
\begin{aligned}
A & =\Gamma^{2}(y-1) y\{45+\Gamma[46+3 \Gamma+y+3 \Gamma y \\
& \left.\left.-(9+7 \Gamma) y^{2}+3(2+\Gamma) y^{3}\right]\right\}-30 \Gamma \ln (1+\Gamma y) \\
B & =3 \Gamma^{2}(\Gamma+1) y\left(-7+10 y-5 y^{3}+2 y^{4}\right) \ln (\Gamma) \\
& +30(1+\Gamma)(1+2 \Gamma) y \ln (1+\Gamma)-30 \ln (1+\Gamma y),(11) \\
C & =-3 \Gamma^{2}(1+\Gamma) y\left[-7+y^{3}(-5+2 y)\right] \ln (\Gamma(1+\Gamma)) \\
& +20 \ln (1+\Gamma y)+10 \Gamma y \ln (\Gamma(1+\Gamma y)) \\
D & =1080 \Gamma^{3}[\Gamma(2+\Gamma)+2(1+\Gamma) \ln (\Gamma)-2(1+\Gamma) \ln (\Gamma(1+\Gamma))]
\end{aligned}
$$

Following (4.19) of Ref. [15], the function $F(\Gamma)$ is then

$$
F(\Gamma)=\frac{\Gamma^{2}}{\pi^{2}(2+\Gamma)^{4}} \int_{0}^{1} G_{\Gamma}^{\prime}(y)^{2} \mathrm{~d} y .
$$




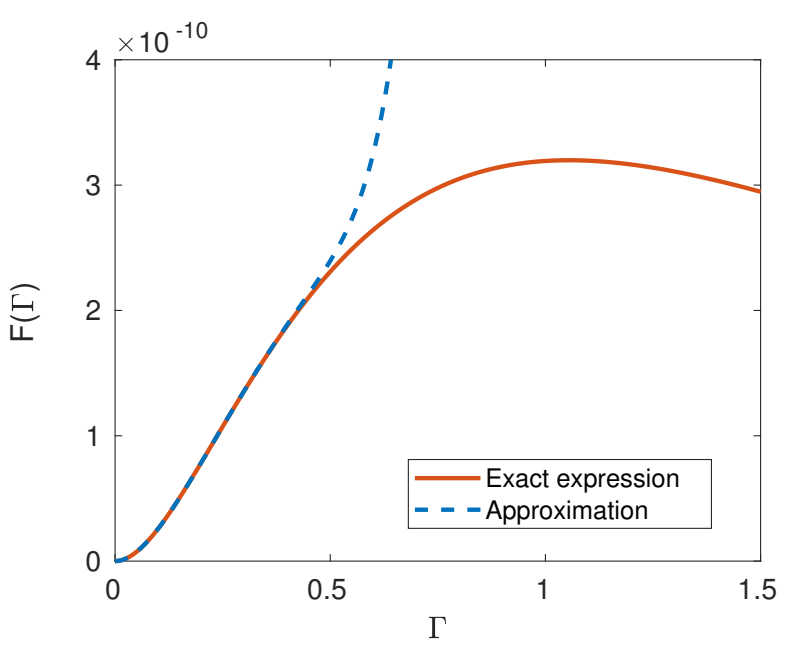

FIG. 5. Function $F(\Gamma)$ associated with cooling by baroclinic streaming and the approximation for $\Gamma<0.4$ used in the present study.
It can be approximated in the range $\Gamma<0.4$ by its 10 -th Taylor polynomial

$$
F(\Gamma) \simeq 10^{-8} \sum_{n=2}^{10} a_{n} \Gamma^{n}
$$

with

$$
\begin{aligned}
& a_{2}=0.3464, a_{3}=-1.039, a_{4}=1.855, a_{5}=-2.57 \\
& a_{6}=3.06, a_{7}=-3.3, a_{8}=3.3, a_{9}=-3.2, a_{10}=3 .
\end{aligned}
$$

Both the exact expression (14) and the approximation (15-16) are plotted in Fig 5.
[1] M. Lighthill, Acoustic Streaming, J. Sound Vib. 61, 391 (1978).

[2] M. Bengtsson and T. Laurell, Ultrasonic Agitation in Microchannels, Anal Bioanal Chem 378, 1716 (2004).

[3] G. G. Yaralioglu, I. O. Wygant, T. C. Marentis, and B. T. Khuri-Yakub, Ultrasonic mixing in microfluidic channels using integrated transducers, Anal. Chem. 76, 3694 (2004).

[4] M. Legay, N. Gondrexon, S. L. Person, P. Boldo, and A. Bontemps, Enhancement of Heat Transfer by Ultrasound: Review and Recent Advances, Intl J. Chem. Engng 2011, 670108 (2011).

[5] B.-G. Loh, S. Hyun, P. I. Ro, and C. Kleinstreuer, Acoustic streaming induced by ultrasonic flexural vibrations and associated enhancement of convective heat transfer, J. Acoust. Soc. Am. 111, 875 (2002).

[6] S. Hyun, D.-R. Mee, and B.-G. Loh, Investigation of convective heat transfer augmentation using acoustic streaming generated by ultrasonic vibrations, Intl J. Heat Mass Transfer 48, 703 (2005).

[7] Y. Lin and B. Farouk, Heat transfer in a rectangular chamber with differentially heated horizontal walls: Effects of a vibrating sidewall, Intl J. Heat Mass Transfer 51, 3179 (2008).

[8] M. Nabavi, K. Siddiqui, and J. Dargahi, Influence of differentially heated horizontal walls on the streaming shape and velocity in a standing wave resonator, Intl J. Heat Mass Transfer 35, 1061 (2008).

[9] M. K. Aktas and T. Ozgumus, The effects of acoustic streaming on thermal convection in an enclosure with differentially heated horizontal walls, Intl J. Heat Mass Transfer 53, 5289 (2010).

[10] T. D. Dreeben and G. P. Chini, Two-dimensional streaming flows in high-intensity discharge lamps, Phys. Fluids 23, 056101 (2011).
[11] G. P. Chini, Z. Malecha, and T. D. Dreeben, Largeamplitude acoustique streaming, J. Fluid Mech. 744, 329 (2014).

[12] J. T. Karlsen, P. Augustsson, and H. Bruus, Acoustic Force Density Acting on Inhomogeneous Fluids in Acoustic Fields, Phys. Rev. Lett. 117, 114504 (2016).

[13] J. T. Karlsen, W. Qiu, P. Augustsson, and H. Bruus, Acoustic Streaming and Its Suppression in Inhomogeneous Fluids, Phys. Rev. Lett. 120, 054501 (2018).

[14] W. Qui, J.H. Joergensen, E. Corato, H. Bruus, and P. Augustsson, Fast Microscale Acoustic Streaming Driven by a Temperature-Gradient-Induced Nondissipative Acoustic Body Force, Phys. Rev. Lett. 127, 064501 (2021).

[15] G. Michel and G. P. Chini, Strong wave-mean-flow coupling in baroclinic acoustic streaming, J. Fluid Mech. 858, 536 (2018).

[16] J. P. Koulakis and S. Putterman, Convective instability in a stratified ideal gas containing an acoustic field, J. Fluid Mech. 915, A25 (2021).

[17] V. Kumar, M. Azharudeen, C. Pothuri, and K. Subramani, Heat transfer mechanism driven by acoustic body force under acoustic fields, Phys. Rev. Fluids 6, 073501 (2021).

[18] P. Vainshtein, M. Fichman, and C. Gutfinger, Acoustic enhancement of heat transfer between two parallel plates, Int. J. Heat Mass Transfer. 38, 1893 (1995).

[19] R. M. Fand and J. Kaye, Acoustic Streaming near a Heated Cylinder, J. Acoust. Soc. Am. 32, 579 (1960).

[20] V. Daru, C. Weisman, D. Baltean-Carlès, and H. Bailliet, Acoustically induced thermal effects on Rayleigh streaming, J. Fluid Mech. 911, A7 (2021).

[21] J. P. Koulakis, S. Pree, A. L. F. Thornton, and S. Putterman, Trapping of plasma enabled by pycnoclinic acoustic force, Phys. Rev. E 98, 043103 (2018). 